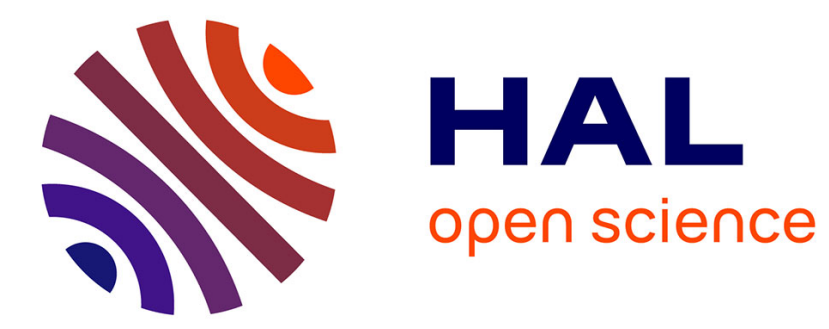

\title{
DES DÉCOUVERTES ET DES CUIVRES. D'UNE FORME DE MONÉTARISATION DU POTLATCH
}

\author{
Marie Mauzé
}

\section{To cite this version:}

Marie Mauzé. DES DÉCOUVERTES ET DES CUIVRES. D'UNE FORME DE MONÉTARISATION DU POTLATCH. Marcel Drach. L'argent, La Découverte, pp.181 - 197, 2004, 9782707143129. halshs01415551

\section{HAL Id: halshs-01415551 \\ https://shs.hal.science/halshs-01415551}

Submitted on 14 Dec 2016

HAL is a multi-disciplinary open access archive for the deposit and dissemination of scientific research documents, whether they are published or not. The documents may come from teaching and research institutions in France or abroad, or from public or private research centers.
L'archive ouverte pluridisciplinaire HAL, est destinée au dépôt et à la diffusion de documents scientifiques de niveau recherche, publiés ou non, émanant des établissements d'enseignement et de recherche français ou étrangers, des laboratoires publics ou privés. 


\section{DES DÉCOUVERTES ET DES CUIVRES. D'UNE FORME DE MONÉTARISATION DU POTLATCH}

Marie Mauzé

\section{Marcel Drach, L'argent}

La Découverte | «Recherches »

2004 | pages 181 à 197

ISBN 9782707143129

Article disponible en ligne à l'adresse :

http://www.cairn.info/l-argent--9782707143129-page-181.htm

\section{!Pour citer cet article :}

Marie Mauzé, «11. Des découvertes et des cuivres. D'une forme de monétarisation du potlatch », in Marcel Drach, L'argent, La Découverte « Recherches », 2004 (), p. 181-197.

Distribution électronique Cairn.info pour La Découverte.

(C) La Découverte. Tous droits réservés pour tous pays.

La reproduction ou représentation de cet article, notamment par photocopie, n'est autorisée que dans les limites des conditions générales d'utilisation du site ou, le cas échéant, des conditions générales de la licence souscrite par votre établissement. Toute autre reproduction ou représentation, en tout ou partie, sous quelque forme et de quelque manière que ce soit, est interdite sauf accord préalable et écrit de l'éditeur, en dehors des cas prévus par la législation en vigueur en France. Il est précisé que son stockage dans une base de données est également interdit. 


\title{
Des découvertes et des cuivres. \\ D'une forme de monétarisation du potlatch
}

\author{
par Marie Mauzé
}

Parler du potlatch comme institution emblématique des sociétés autochtones de la côte Nord-Ouest de l'Amérique du Nord, c'est préciser d'entrée de jeu qu'il s'agit là d'une institution coloniale, née dans la seconde moitié du XIX ${ }^{\mathrm{e}}$ siècle, et qui a quasiment disparu dans les années 1930, à la suite de peines d'emprisonnement prononcées à l'encontre de chefs kwakiutl et de la confiscation d'objets cérémoniels, dans le cadre de l'application de la loi dite « anti-potlatch » de 1884, qui interdisait une institution considérée par les agents gouvernementaux et les missionnaires comme une « coutume aberrante, caractérisée par des dépenses excessives, et démoralisante » [Fisher, 1977, p. 206]. Le terme " potlatch » lui-même n'appartient à aucune langue indigène de la région, il est emprunté au sabir chinook, langue de traite en usage sur la côte Ouest du Pacifique au XIX ${ }^{\mathrm{e}}$ siècle ; probablement apparu dans les années 1860, il signifie « don » ou « action de donner »; on lui reconnaît une parenté linguistique avec le mot nootka " pachitle ${ }^{1}$ », utilisé pour des dons dans des échanges cérémoniels [Mauzé, 1986, p. 27, 42, voir aussi Goldman, 1975, p. 131, 245 ; Wolf, 1999, p. 111-112]. Sous un même vocable ont été regroupées des appellations concernant différentes cérémonies au cours desquelles étaient distribués des biens. Pour les représentants de l'administration coloniale, le potlatch était un obstacle au progrès et à l'entreprise de civilisation des populations indiennes. Pour les Indiens, sa pratique était faire acte de résistance aux autorités ; elle est aujourd'hui un témoignage de revendication identitaire [Mauzé, 1995].

Avec Franz Boas, le «potlatch » devient un concept majeur de l'anthropologie [Mauzé, 1996], l'institution correspondante pouvant être analysée sous de multiples aspects : économiques, psychologiques, religieux, etc. [Schulte-Tenckhoff,

1. On doit l'une des premières descriptions d'un potlatch à l'explorateur Robert Brown en 1864. L'auteur utilise le terme « pacheetl » [Hayman, 1989, p. 165]. 
1986 ; Masco, 1995]. Il revient à Marcel Mauss [1925] d'avoir analysé le potlatch comme un système de « prestations totales » engageant des groupes et des personnes, pour autant que ces dernières (exemplairement les chefs) représentent ces groupes, le don relevant de diverses déterminations évoquées ci-dessus. Pour Mauss, le potlatch est inséparable de trois obligations : donner, recevoir, rendre. Présenté sous sa forme la plus générale, il est un système d'accumulation et de distribution de biens au moyen duquel un chef valide par des dons l'accession à la dignité à laquelle il est candidat [Barnett, 1968]. La distribution de biens est l'acte qui fonde la reconnaissance magico-religieuse et politico-sociale des statuts et reconduit la validité d'une hiérarchie des statuts, déterminée par la capacité qu'offre le nom ou le titre associé à un statut d'accéder à la maîtrise de pouvoirs surnaturels qui lui sont attachés ${ }^{2}$. La validation d'un statut et de privilèges n'est pas séparable, à partir du dernier quart du XIX ${ }^{\mathrm{e}}$ siècle, de l'affirmation d'une victoire acquise lors d'une compétition pouvant aller jusqu'à la manifestation d'une rivalité exacerbée. On a là en germe une dérive de la pratique du potlatch qui se manifeste essentiellement chez les Kwakiutl ; c'est l'expression qu'a choisi d'en retenir l'anthropologie française à la suite de Marcel Mauss, pour qui le potlatch est un « un système de prestations totales de type agonistique » [Mauss, 19253].

Si elle s'appuie sur des textes désormais classiques, l'interprétation de Mauss, contrairement à ce qu' affirme Maurice Godelier [1999], n'en fait pas moins généralement l'impasse sur le contexte historique dans lequel s'est développée cette « guerre des richesses » devenue nécessaire pour accéder à des positions au sein de la hiérarchie structurant l'organisation des groupes. La situation coloniale a suscité l'apparition de contextes nouveaux qui ont, sinon modifié en profondeur les structures sociales et les logiques culturelles de ces sociétés, du moins créé des tensions au sein de ces structures et de ces logiques, et donné naissance à des réaménagements et des innovations, qui sont autant d'échos de changements intervenus dans le monde indigène. Si l'on considère, pour les Kwakiutl, la période allant des années 1850 jusqu'aux années 1920, appelée par Helen Codere [1950] la «Potlatch Period », en opposition à celle qui précède («Pre-Potlatch Period », 1774-1849), on remarque que la fréquence des potlatchs s'accélère en même temps qu' augmente la quantité des biens distribués ${ }^{4}$ et que s'élargit la sphère des groupes concernés par les échanges : on parle alors de grands potlatchs « inter-tribaux ». C'est l'insertion de la population autochtone dans l'économie de marché qui

2. Selon Goldman [1975, p. 181], « ce que les observateurs ont considéré comme l'expression d'une rivalité à propos de l'acquisition d'un rang, sont des démonstrations individuelles de pouvoirs surnaturels, ces démonstrations se manifestant sous la forme d'une hostilité agressive envers les rivaux pour montrer que la capacité de destruction est un des attributs de ce pouvoir »; voir aussi Wolf [1999, p. 116]

3. Voir aussi une critique de l'interprétation de Georges Bataille in [Mauzé, 1987].

4. Hélène Codere $[1950,1961]$ a rassemblé des données relatives à la nature et la quantité des biens distribués dans les potlatch kwakiutl. Au début du XIX ${ }^{\mathrm{e}}$ siècle, la quantité de biens distribués n'excédait pas 300 couvertures ou 50 peaux tannées ; en 1869, 9000 couvertures sont distribuées, en 1895, 13000. 
permet un enrichissement rapide des Indiens soit par la traite des fourrures, soit à partir des années 1870 par le travail dans les industries de la pêche et du bois [Knight, 1978]. L'introduction des couvertures de la Compagnie de la baie d'Hudson a eu un impact important sur le volume des biens échangés. Quel que soit leur statut, les intéressés - chefs, nobles ou gens du commun -, tout le monde pouvait acquérir des couvertures auprès des traiteurs. L'obtention de biens matériels était à la portée de chacun sans que soit mis en jeu le statut des personnes par rapport à un ordre symbolique qui continuait de régir les relations entre membres d'un même groupe. Nous avons là un phénomène qui ne peut être analysé qu'au regard de la « rencontre coloniale », marquée par l'influence directe de l'économie capitaliste sur l'économie traditionnelle, mais aussi par la dramatique diminution de la population et par l'arrêt des guerres et des raids de capture d'esclaves ${ }^{5}$ [Codere, 1950, 1961 ; Drucker et Heizer, 1967 ; Mauzé, 1986 ; Boyd, 1999]. C'est aussi dans le dernier quart du XIX ${ }^{\mathrm{e}}$ siècle que les peuples de la côte Nord-Ouest passent sous la tutelle des gouvernements canadien et américain, dans le même temps où l'action des missionnaires étend son influence sur les communautés autochtones, on a là autant de facteurs qui perturbent la vie économique, politique et religieuse des Indiens ${ }^{6}$.

\section{POTLATCH ET COLONISATION}

La période considérée nous confronte à une situation apparemment paradoxale. En effet, la diminution de la population entraîne la vacance d'un certain nombre de positions au sein du système hiérarchique, directement articulé au système cosmologique, les chefs étant censés réincarner les ancêtres fondateurs des groupes. Traditionnellement ces positions ne pouvaient être acquises que par des titulaires dont la légitimité de la revendication était fondée sur le rang de naissance. Or il arrive un moment où, dans certains cas, la reconduction des offices n'est plus possible. C'est dans ce contexte que se manifestent des stratégies nouvelles d'acquisition de statuts, mises en œuvre par des distributions de biens massives émanant de candidats à la légitimité discutable : femmes qui, dans l'ordre ancien, ne pouvaient prétendre détenir des positions de haut $\operatorname{rang}^{7}$; nobles de rang inférieur

5. La guerre était un moyen d'acquérir des biens matériels (peaux, fourrures, esclaves, produits alimentaires) et immatériels. Chez les Kwakiutl, par exemple, la guerre avait pour but la captation de biens symboliques, le guerrier qui tuait un ennemi s'appropriait certains de ses privilèges, notamment ceux qui avaient trait à la détention de danses dans les sociétés secrètes [Boas, 1897, p. 424 ; Boas et Hunt 1905, p. 223 ; Boas, 1921, p. 1016-1017, voir aussi Mauzé, 1986, p. 24 ; Masco, 1995, p. 50-51].

6. Masco [1995] propose une excellente analyse de l'impact de la colonisation sur l'ordre cosmologique et le développement consécutif de rituels destinés à régénérer un ordre désormais en péril.

7. Dans le cas où une femme est l'aînée de la lignée, elle peut succéder à son père, mais temporairement. Elle acquiert le statut d'un homme par le nom qu'elle reçoit, nom qu'elle doit transmettre à son fils quand celui-ci est en âge de l'assumer [Boas, 1966, p. 52]. 
et gens du commun qui parviennent désormais à trouver place au sein de la hiérarchie de leur groupe, notamment en acquérant des privilèges par le mariage [Drucker et Heizer, 1967 ; Mauzé, 1986]. En raison de la possibilité qui leur était ainsi offerte - un gendre pouvait acquérir des privilèges par l'intermédiaire de son épouse pour transmission aux enfants du couple -, les Kwakiutl, plus que les membres des autres sociétés de la côte Nord-Ouest, ont su jouer des règles de filiation et d'alliance pour maximiser la valeur de leur patrimoine symbolique, tout en prenant avantage de l'afflux de biens nouveaux qui irriguait l'économie traditionnelle. L'organisation sociale s'est maintenue tout en s'adaptant aux contraintes de la situation coloniale.

Immatériels et matériels, les biens prestigieux circulent dans les potlatchs. Les biens immatériels comprennent des noms, des blasons, des chants et des danses ; on distingue les biens inaliénables [Weiner 1985, 1992] qui fondent l'identité du groupe et par conséquent circulent à l'intérieur d'un même groupe, et les biens aliénables, notamment les noms et les danses relevant de la sphère d'activité des sociétés secrètes ; ceux-ci ont vocation à passer d'un groupe à un autre. Au nombre des biens matériels, on compte les coquillages d'haliotide, les peaux tannées, les fourrures de loutre de mer, les canoës, les esclaves, mais aussi les cuivres, les couvertures chilkat blasonnées, les masques, les plats, les coffres, etc. La circulation de biens hautement valorisés tient une place éminente dans les rites funéraires - notamment chez les Tlingit, les Haida et les Tsimshian -, dans les cérémonies de dation de nom ou encore les mariages. Les cuivres, qui chez les Haida et les Tsimshian sont la propriété du groupe, sont transmis chez les Kwakiutl au gendre par le beau-père au titre soit de la dot soit du remboursement de la compensation matrimoniale ${ }^{8}$. Parmi les biens matériels, une distinction est opérée entre les objets qui portent des noms (masques, cuivres, plats, canoës) ${ }^{9}$, et ceux qui n'en portent pas : fourrures, peaux, esclaves. Alors que les premiers induisent une relation marquée entre ceux qui donnent et ceux qui reçoivent, les seconds constituent « une classe d'objets possédant une valeur d'échange généralisée contre des biens et des services » [Nash, 1966 in Harkin, 1997, p. 130, voir aussi Harkin, p. 127].

Ces choses de valeur constituent moins un capital matériel que l'expression d'une puissance magico-religieuse du fait qu'elles sont considérées comme des " contenant $\mathrm{s}^{10}$ » chargés de pouvoirs surnaturels et de force vitale donnés aux humains par les ancêtres et les esprits. Tout se passe comme si les attributs conférés aux choses fournissaient la preuve que les chefs, en raison de la relation qui

8. Il est des cas où des biens inaliénables sont devenus aliénables en raison des changements qui se sont produits à la fin du XIX ${ }^{\mathrm{e}}$ siècle. Il semblerait que les cuivres en soient une des illustrations.

9. Les masques, les plats, les coffres figurent généralement des blasons et sont l'expression matérielle d'un privilège.

10. Les peaux en tant qu'elles forment une enveloppe peuvent être aussi considérées comme les contenants d'énergie vitale relevant du domaine animal [Walens, 1981, p. 150-153]. 
les attache à un nom, étaient investis de certaines formes de pouvoir acquises auprès d'êtres puissants, disposant de la capacité à maîtriser les échanges entre le monde humain et le monde non humain [Harkin, 1997, p. 127], d'accumuler des richesses et de les distribuer. La distribution de biens offre aux chefs l'occasion non seulement de faire montre de leur pouvoir mais aussi de le renforcer en transférant des forces vitales aux représentants des autres groupes [Wolf, 1999, p. 120], ce qu'exprime un langage métaphorique ayant trait à l'ingurgitation.

Un fait marquant a été l'introduction de la couverture de la Compagnie de la baie d'Hudson, dont la mise en circulation va affecter une économie indienne fondée sur le troc et sur la réciprocité des échanges, dans la mesure où les réserves européennes de couvertures sont illimitées. Au titre de moyen d'échange généralisé, la couverture n'occupe pas une place jusqu'alors inconnue. Réappropriée dans le cadre de la logique culturelle autochtone, elle opère comme substitut de biens désormais abandonnés comme les esclaves, les fourrures ou les peaux tannées $^{11}$ : considérée comme «l'enveloppe symbolique du Blanc ${ }^{12}$ », elle devient « un nouveau standard de pouvoir des chefs, car le Blanc précisément représentait le pouvoir » [Kobrinsky, 1975, p. 43 $3^{13}$ ]. C'est ainsi que les biens d'origine européenne sont envisagés comme des biens ayant une valeur dispensée par les dieux, et sont incorporés aux systèmes d'échange cérémoniel [Sahlins, 1988, p. 6]. Après un siècle de contact entre les Indiens et les Européens, et en dépit de l'afflux de produits manufacturés, les transferts de biens continuent de s'opérer « dans les formes solennelles du potlatch » [1950 (1925), p. 194].

La couverture devient l'instrument de mesure adéquat pour évaluer le «poids » des noms des chefs, à ceci près que cette évaluation ne s'effectue pas à l'aune de la nature et de la qualité des objets distribués, comme autrefois, mais à celle de leur quantité. Comme l'a souligné Marshall Sahlins [1988, 1999], il est remarquable que les chefs des sociétés de la côte Nord-Ouest aient très rapidement choisi d'acquérir, «par dizaines de milliers », « des produits standardisés en fixant leur choix sur des couvertures en laine », alors par exemple que les chefs hawaïens préféraient acquérir des produits de luxe. «Et plutôt que d'accumuler [leurs propres biens] comme le faisaient les chefs hawaïens, poursuit Sahlins, les Kwakiutl distribuaient leurs couvertures de façon à pouvoir affirmer leurs droits au pouvoir

11. Les fourrures et les peaux tannées sont offertes aux nobles tandis que les tapis en écorce de cèdre vont aux gens du commun, conformément aux représentations d'une hiérarchie instituée entre le règne animal et le règne végétal [voir Boas, 1921, p. 878-883]. Voir aussi [Sewid-Smith, 1986, p. 63].

12. Ce qui différencie les hommes des animaux, c'est leur aspect extérieur. Sous leur enveloppe, animaux et humains partagent une même substance. Ancêtres et esprits quittent leur apparence première en se débarrassant de leur dépouille animale quand ils agissent parmi les hommes.

13. Il faut comprendre que le «pouvoir » dont il s'agit ici n'est pas le «pouvoir colonial » exercé sur les Indiens, mais le « pouvoir » tel qu'il est envisagé par les Indiens de la côte Nord-Ouest, en tant qu'il a une origine extérieure à la société. Posséder des biens apportés par les Blancs constitue une certaine forme de pouvoir car cela implique une relation avec des êtres venus de l'extérieur, du lointain [Harkin, 1997, p. 131]. 
[...]. Au contraire des Kwakiutl qui taillaient leur prééminence dans de la toile ordinaire, l'élite hawaïenne se concentrait sur des projets uniques d'accroissement économique. Il est vrai que les chefs hawaïens étaient tous des descendants plus ou moins proches des dieux, et que leur souci principal était de transformer des différences quantitatives de la généalogie en distinctions qualitatives de statut social. » Mais, poursuit Sahlins, « les chefs kwakiutl représentaient déjà des lignées distinctes et non apparentées [entre elles] avec des origines et des pouvoirs bien [différenciés]. En tant qu'héritiers de patrimoines et d'ancêtres uniques, ils utilisaient des marchandises européennes courantes pour transformer [des] différence[s] qualitatives de généalogies en [des] mesures quantitatives du [statut] social » [Sahlins, 1988, p. 41-42; 1999, p. 19, voir aussi Kobrinski, 1975, p. 51]. Le «poids » des noms est ainsi matérialisé sous la forme de piles de couvertures dont le volume est un indicateur de la différenciation de statuts. On note encore qu'en faisant le choix d'introduire dans les échanges non pas la monnaie britannique puis canadienne, mais une marchandise dotée d'une valeur symbolique équivalente à celle conférée à certains biens d'origine indigène, les peuples de la côte Nord-Ouest ont maintenu une séparation entre une économie de profit dans le cadre de laquelle travail et services sont évalués en argent, et une économie traditionnelle pour laquelle certains produits manufacturés (les couvertures, par exemple), acquis par le biais de l'intégration dans l'économie marchande, sont utilisés pour valider des pouvoirs relevant de la sphère du mythe et de la religion.

Devenues des objets de don, les couvertures permettent l'évaluation d'autres biens de prestige. Une différence notable doit cependant être soulignée entre le mode d'acquisition des produits d'origine indigène et celui des produits manufacturés dont l'obtention ne faisait pas l'objet de procédures rituelles spécifiques : en clair, il suffisait pour obtenir les seconds d'en avoir les moyens. On est là dans une situation où sont mis en parallèle pour un temps deux régimes de valeur : l'un ancré dans une économie symbolique fondée sur une circulation imaginaire de forces vitales obtenues des dieux et des ancêtres, l'autre directement articulé à une économie extérieure dont des produits sont devenus des biens prestigieux [Wolf, 1999, p. 122].

\section{LE CUIVRE : UN OBJET PRÉCIEUX}

Ce que l'on dénomme sous le mot « cuivre » est une plaque de cuivre ouvragée dont la forme est généralement comparée à celle d'un bouclier. Cette forme aurait probablement été inventée par les Tlingit [Duff, 1981], société à partir de laquelle les cuivres se sont diffusés chez les groupes voisins. Certains auteurs comme T. Waterman [1923, p. 450] ont émis l'hypothèse que la forme du cuivre serait dérivée du masque Qonaqadet, la partie supérieure de l'objet figurant la tête du monstre marin ou seulement son front. Tout en prenant en compte cette 
hypothèse, Lévi-Strauss [1979] établit un parallèle du point de vue de la forme et de la fonction entre le masque Swaihwé des Salish et le cuivre des Kwakiutl ${ }^{14}$.

On sait peu de chose de l'origine des cuivres. Le cuivre-objet n'a pas été décrit par les premiers voyageurs qui ont visité la région à compter du dernier quart du XVIII ${ }^{e}$ siècle ; la première mention qui en est faite est due au capitaine Lisiansky, lequel rapporte avoir vu en 1804 un objet « curieux », dérobé par le comte Baranov dans la maison d'un chef tlingit après la mise à sac par les Russes des habitations du village de Sitka [Lisiansky, 1814, p. 150 ; Emmons, 1991, p. 180-181]. L'existence des cuivres est bien attestée au début du XIX ${ }^{\mathrm{e}}$ siècle. Les observateurs notent que les Tlingit (Chilkat) possèdent un cuivre natif ${ }^{15}$ qu' ils façonnent en forme de bouclier d'environ deux pieds de hauteur ; les cuivres sont décorés de motifs représentant des humains ou des animaux [Keithan, 1964, p. 67].

Très tôt, quelques années après le contact, le métal natif a cédé la place au cuivre feuillard. Une quinzaine d'années après la découverte de la région par les Espagnols, le marché était quasiment saturé. Les cuivres que l'on trouve dans les collections américaines et européennes sont en cuivre feuillard [Keithan, 1964, p. 67]. Il importe de noter que les sociétés de la côte Nord-Ouest ont d'emblée assigné au métal importé les qualités réelles ou symboliques qu'ils attribuaient au métal natif. Le cuivre est ainsi une « chose » dont la nature ne réside pas seulement dans les caractères ou les vertus du matériau, mais aussi dans la charge symbolique dont elle est porteuse. Il est doté d'une vie autonome propre « soit à produire de nouveaux rapports sociaux, soit à en reproduire d'anciens » [Godelier, 1996, p. 94]. Dans la mythologie, le cuivre-objet est associé au soleil ou à la lumière [Boas et Hunt, 1905, p. 45 ; Goldman, 1975, p. 226 ; voir aussi Mauss, 1950, (1925), p. $222^{16}$ ] ; il est identifié au saumon, symbole de richesse du monde marin

14. Sur la base de rapprochements linguistiques, Keithan [1967, p. 74-75] émet l'hypothèse que la forme du cuivre correspondrait à celle des pointes de flèche, au changement d'échelle près. La ressemblance entre le masque Swaihwé et le cuivre a déjà été notée par Wingert [1949]. Lévi-Strauss souligne : «Si chez les Salish, le Swaihwé est le moyen d'acquérir la richesse, nous croyons avoir établi que chez les Kwakiutl, les cuivres - richesses suprêmes - sont la métaphore du Swaihwé » [1979, p. 112116]. Voir aussi l'analyse de MacDonald [1981] qui considère que le cuivre renvoie à une expression abstraite de la richesse associée aux prestations de mariage et à une représentation du monde cosmologique, telle qu'elle apparaît notamment sur les frontons de maison.

15. Le cuivre natif provenait des rivières Copper et White [Emmons, 1991, p. 177 ; Duff, 1981]. Il existait sous forme de petits lingots que l'on pouvait ramasser dans le lit des rivières. Les Tlingit ont obtenu le cuivre des Atna (de la rivière du cuivre) qui leur auraient enseigné la technique de martelage en feuille du lingot à l'aide d'un marteau en pierre [Krause, 1956, p. 148 ; Keithan, 1964, p. 71 ; Emmons, 1991, p. 178]. Les Tlingit ont à leur tour échangé les feuilles de cuivre avec leurs voisins, les Haida et les Tsimshian, qui a leur tour les ont troquées avec les Kwakiutl. Le cuivre natif était utilisé sur la côte Nord-Ouest depuis au moins 2000 ans [MacDonald, 1996] ; il servait à fabriquer des ornements (colliers, bracelets) pour les nobles mais aussi des armes : pointes de flèche, lances, poignards et plastrons destinés à protéger les guerriers [Emmons, 1991, p. 175, 176, 180, 375].

16. À la couleur rouge est associé un registre symbolique très large : la vie, le surnaturel, la richesse, etc. Voir par exemple [Reid, 1986, p. 76-77]. 
dont il porte parfois le nom et dont il partage l'odeur [Mauss, ibid.; de WiderspachThor, 1981, p. 159]. C'est un objet rare, réputé avoir une origine lointaine, qu'elle soit céleste ${ }^{17}$, aquatique ou chtonienne. En tout cas, il vient d'un au-delà du monde humain et c'est précisément de cette origine mystérieuse qu'il tient son caractère de support de pouvoir et de symbole de richesse ${ }^{18}$, matérialisé par son éclat.

Plutôt que des substituts d'esprits surnaturels ou d'ancêtres, les cuivres apparaissent comme la manifestation tangible d'une relation qu'entretient une certaine catégorie d'humains - les chefs et les nobles - avec les êtres surnaturels ; ils sont en quelque sorte des contenants d'un pouvoir qui vient des esprits. En ce sens, il y a indistinction entre pouvoir et richesse parce que les deux ont une même origine. Mais le cuivre ne se situerait-il pas d'un autre point de vue à l'articulation du monde non humain et du monde humain ? En effet, le cuivre est à la fois métaphore et substitut du corps humain, comme le montre le vocabulaire désignant les différentes parties de l'objet et la fonction attribuée à chacune de ces parties.

L'objet est constitué de deux parties principales : une partie supérieure dont les bords s'évasent vers le haut et une partie inférieure que mettent en évidence deux arêtes perpendiculaires [Niblack, 1890, p. 335-336 ; Lévi-Strauss, 1979, p. 133-134]. La partie supérieure est appelée d'un terme qui désigne le visage, notamment quand elle représente un animal-blason vu de face [Emmons, 1991, p. 179 ; Olson, 1933, p. 54 ; Boas, 1966, p. 82 ; Kan, 1989, p. 41] ; du reste, le nom ${ }^{19}$ du cuivre est souvent dérivé du nom de l'animal qui est figuré. Le cuivre comprend une partie inférieure qui correspond au reste du corps ; formant un $\mathrm{T}$, les arêtes perpendiculaires, martelées en creux, en constituent la « colonne vertébrale ». On trouverait d'autres assimilations des parties du cuivre à des parties du corps, que ce soit chez les Kwakiutl ou chez les Tlingit ${ }^{20}$.

Outre les traits morphologiques qui l'apparentent à un corps humain, le cuivre est un être vivant : il parle, il grogne, il exprime des sentiments, des sensations et des besoins. Il peut demander à être donné ou à être détruit, il peut avoir froid et

17. Les Tlingit, rappelle Claude Lévi-Strauss [1989, p. 109], rattachent l'éclat resplendissant du cuivre à son origine céleste.

18. Cette relation du cuivre à la richesse est fondamentale. Le cuivre est ce par quoi la richesse advient à moins qu'il ne soit la richesse par excellence. Les humains ont obtenu le cuivre de personnages surnaturels qu'ils ont eu le bonheur de rencontrer sur leur chemin, tel Qonaqadet, monstre des marées des Tlingit, ou Qomoqwa, esprit marin des Kwakiutl appelé aussi « Faiseur de Richesses » [Kan, 1989 ; Boas, 1966, p. 303 ; Lévi-Strauss, 1979 ; MacDonald, 1996].

19. Les noms des cuivres relèvent de différentes catégories : certains ont à voir avec l'animal peint ou gravé sur sa partie supérieure qui représente généralement l'ancêtre du groupe ; d'autres ont une connotation guerrière ou font référence à la richesse ou encore à la valeur « économique » de l'objet [Boas, 1897, p. 344 ; Hunt, 1923 ; Drucker et Heizer, 1967, p. 127].

20. Chez les Kwakiutl, deux termes sont utilisés pour définir la partie inférieure ; l'un renvoie aux côtes et par extension à la cage thoracique, l'autre au squelette [de Widerspach-Thor, 1981, p. 166]. Les Tlingit distinguent l'arête verticale ou « colonne vertébrale » de l'arête horizontale ou «épaules » [Kan, 1989, p. 241]. 
faim [1950 (1925), p. 225]. «Briser un cuivre, c'est le tuer », écrit Goldman [1975, p. 150] ; « Jeter un cuivre à l'eau, c'est le noyer », écrit Boas [1897, p. 564]. Le cuivre éprouve la différence entre la vie et la mort. Mort, il peut être réanimé à la condition que sa « colonne vertébrale » soit intacte. La « réanimation » du cuivre fait l'objet d'une procédure simple ; il suffit en effet de riveter sur sa partie centrale $^{21}$ un ou plusieurs fragments d'un autre cuivre : ce retour à la vie restitue au cuivre sa vocation à circuler dans les échanges cérémoniels.

Ainsi n'est-il pas inapproprié de parler de l'immortalité du cuivre et de considérer l'existence d'une collectivité de ces êtres immortels dont les apparitions, les disparitions, les transformations obéissent à des lois qui leur sont propres, même si elles ménagent une place à l'intervention humaine. Il y a plus, et l'on retrouve la relation déjà évoquée entre pouvoir et richesse. La frontière toujours incertaine entre monde humain et monde non humain s'estompe lorsque l'on considère les rapports qu'entretiennent les chefs et les cuivres ; tout se passe en effet comme si les uns et les autres participaient d'une même substance qui serait leur partage et les vouerait ensemble à une immortalité commune dont le cuivre serait cependant la manifestation exemplaire. À la mort d'un chef, son cuivre est découpé en morceaux. Ces fragments, appelés « os du chef », sont répartis entre les chefs invités au potlatch organisé à la mémoire du défunt [Drucker et Heizer, 1967, p. 70 ; Garfield, 1939, p. 238 ; Harkin, 1997, p. 129 ; McIlwraith, 1992, p. 225 ; Kan, 1989, p. 242]. Tout indique qu'il y a lieu de prendre l'expression « os du chef » à la lettre. Si le cuivre est animé, s'il peut être réduit à un résidu matériel constitué par les os du défunt, alors le cuivre est un chef ou plus exactement cuivres et chefs participent d'une même identité. Nous demeurons dans le même registre de substitution lorsque le cuivre est assimilé à l'esclave, c'est-à-dire à une propriété du chef qui vaut marque de son prestige. Les esclaves étaient sacrifiés à la mort de leur maitre, lors des rituels d'initiation ou encore à l'occasion de l'érection de mâts funéraires. Les Tsimshian disent que le cuivre est le sang coagulé de l'esclave mis à mort [Duff, 195922]. C'est après qu'a été interdite la mise à mort des esclaves, au milieu du XIX' siècle, que les cuivres leur ont été substitués, à ceci près que l'accompagnement du chef jusqu'au pays des morts a été remplacé par la présentation de cuivres sur la tombe et que l'ensevelissement du corps de l'esclave dans la fosse où était érigé le mât funéraire a été remplacé par la présence d'un cuivre placé à la base du mât [Boas, 1897, p. 439-440 ; 1921, p. 541 ; de Laguna, 1972, p. 470-471 ; Garfield, 1939, p. 274 ; Donald, 1997, p. 273]. Sur ces transformations, Martine de WiderspachThor [1981, p. 170] fait une remarque éclairante : l'utilisation, dans les rituels kwakiutl dits cannibales, de cuivres en place des corps humains a permis de sauvegarder un rituel sans en changer le contenu, en replaçant le corps humain par le

21. Selon George Hunt [1923], la forme en T était considérée par les Kwakiutl comme le « cœur » du cuivre où se logeait sa force.

22. Je remercie Margaret Seguin de m'avoir signalé cette référence. 
cuivre qui le représentait ${ }^{23}$. En raison de sa forte charge symbolique, le cuivre représente l'objet précieux par excellence dans le système des échanges cérémoniels. Il circulait à travers les frontières qui séparaient les différentes sphères du cosmos et les différents groupes sociaux [Harkin, 1997, p. 129 ; Wolf, 1999, p. 122]

\section{LA VALEUR DES CUIVRES}

Comme on l'a vu, les cuivres sont exhibés lors de cérémonies de dation de nom ou de mariage ${ }^{24}$ et dans le cadre de certains rituels ; ils sont découpés en morceaux dans des potlatchs funéraires ou encore brisés dans des potlatchs de rivalité, voire complètement détruits pour humilier un rival ou laver une insulte [Duff, 1981]. Les cuivres peuvent être achetés, vendus ou échangés contre d'autres marchandises. Chez les Tlingit, ils servent de moyen de paiement en vue de l'acquisition de droits de pêche [de Laguna, 1972, p. 354]. Donnés par le beau-père au gendre à titre de paiement du prix de la fiancée ou de remboursement de la compensation matrimoniale, ils constituent un capital important pour le gendre.

Autour du cuivre, ont lieu des opérations complexes qui mettent concurremment en jeu différents niveaux de représentations appartenant aussi bien au monde autochtone qu'au monde euro-canadien. Nous ne reviendrons pas sur la qualité d'objet précieux du cuivre, qu'il conserve lorsqu'il est transmis au sein du groupe ; parallèlement, le cuivre a une valeur marchande quand il fait l'objet d'une transaction « économique » lors d'une cession d'un groupe à un autre. De bien précieux, il peut donc devenir une « marchandise » en tant qu'il est une chose que l'on obtient contre paiement, mais cet état temporaire est réversible quand il fait l'objet d'un don avec obligation de contre-don. Le cuivre a un rang quand il fait l'objet d'un don ; il a un prix quand il est une marchandise ${ }^{25}$.

Les cuivres ont statut de proto-unités de compte ${ }^{26}$ valorisant les échanges. Comme on l'a vu, leur valeur d'échange peut être exprimée en canoës, en esclaves, en peaux tannées et, depuis la seconde moitié du XIX ${ }^{e}$ siècle, en couvertures. La valeur du cuivre dépend de sa taille ${ }^{27}$, de l'épaisseur de la pièce de métal à partir

23. Le cuivre remplace le cadavre ou le corps momifié qui est la nourriture de l'initié (de WiderspachThor, ibid.). Chez les Kwakiutl, les ennemis tombés au combat sont décapités, les trophées sont fichés sur des pieux dans le village des vainqueurs. Les têtes ont été remplacées par des cuivres en bois [Boas, 1966, p. 104-105].

24. Dans certains groupes kwakiutl, le canöe occupe la même place symbolique et sémantique que les cuivres [Mauzé, 1989, p. 119].

25. J'emprunte cette idée à C. A. Gregory [1982] ; en effet, selon cet auteur, les marchandises ont des prix, les dons ont un rang.

26. Je dois l'emploi de cette expression à Marcel Drach.

27. L'afflux de métal importé - le cuivre natif était une denrée rare - entrâna la baisse de la valeur des cuivres, mais eut pour conséquence d'en augmenter la taille. 
de laquelle il a été façonné, de sa sonoritéé ${ }^{28}$. Au sein des groupes septentrionaux (Tlingit et Haida), un cuivre pouvait valoir, dans les années 1840, entre cinq et dix esclaves, valeur qui pouvait être augmentée dans certains cas d'un canoë ou de boîtes de graisse de poisson-chandelle [Donald, 1997, p. 150, 151] ; il pouvait atteindre l'équivalent de plusieurs milliers de couvertures à la fin du XIXe siècle. La valeur d'un cuivre s'accroît à chaque fois qu'il change de main. Cependant, comme le montrent les données ethnographiques, il faut nuancer l'idée trop souvent répandue que la valeur d'un cuivre double à chaque changement de propriétaire [voir par exemple Hunt, 1923]. La valeur du cuivre dépend aussi de son pedigree, établi à partir de l'identité ou de la qualité sociale de son propriétaire ou de ses propriétaires successifs et des différentes fonctions qu'il a assumées au cours de sa « vie sociale ${ }^{29} »$.

La vente d'un cuivre revêt un caractère très formel [Garfield, 1939 ; Boas, 1897]. La procédure est placée sous le contrôle de « tally men », chargés de rassembler et de compter le nombre de couvertures qui établissait le montant ${ }^{30} ;$ plusieurs étapes rythmaient la cérémonie de la vente, chacune correspondant à la montée des enchères [Boas, 1897, p. 345]. En vérité, le « prix » d'un cuivre est évalué en fonction de sa renommée et de celle de son détenteur, mais aussi de façon plus pragmatique en fonction des circonstances qui président à la nécessité de sa vente [Boas, 1897, p. 344]. Le cuivre agit là comme capital qui ne sera pas thésaurisé, mais, par le transfert de son équivalent en couvertures, sera converti en statut. Nécessité faisant loi, il arrive qu'un cuivre soit vendu à bon prix, c'est-àdire pour un montant moins élevé que son prix d'achat, notamment quand il s'agit d'organiser un potlatch funéraire dans les quelques années suivant le décès d'un chef. Ce type de transaction n'entraîne nul déshonneur ${ }^{31}$. Cependant, un jeune chef ayant reçu un cuivre de son beau-père a intérêt à faire monter les enchères dans le but de réunir le plus grand nombre de couvertures et ainsi affirmer son rang.

28. Les sociétés de la côte Nord-Ouest ont établi une distinction entre les « vrais » cuivres, en métal natif et les «faux » cuivres, en métal importé. Les Kwakiutl utilisent les expressions : «White Man Copper » ou « Smooth Face » [Hunt, 1923] pour dénommer les faux cuivres. En vérité, cette distinction entre vrais et faux cuivres est devenue rapidement fictive, car depuis le début du XIX $X^{\mathrm{e}}$ siècle tous les cuivres étaient fabriqués à partir de cuivre feuillard. La pureté du métal était mesurée en fonction du son que produisait le cuivre lorsqu'il était frappé. Un vrai cuivre devait avoir une sonorité sourde [Emmons, 1991, p. 180, 181 ; Hunt, 1923].

29. L'expression « vie sociale » d'un objet est empruntée à Arjun Appadurai [1986].

30. Les Kwakiutl utilisaient des instruments-étalons tels que les bâtons ou les pierres pour mesurer le nombre de couvertures : 1 bâton équivalait à 150 couvertures, une pierre à 10 [Hunt, 1923].

31. Les Kwakiutl désignaient ce type de transaction par l'expression «Tenir le cuivre la tête en bas » [Drucker et Heizer, 1967, p. 77]. 
Mauss a parlé à propos du cuivre de «monnaie de renommée », en désignant ainsi une monnaie-talisman dont l'utilisation est circonscrite aux échanges de clan à clan ou de tribu à tribu [Mauss, 1950 (1925), p. 221], et « dont la possession conférait à leur détenteur un pouvoir qui devint aisément un pouvoir d'achat [...] employé [non pas] à l'acquisition des moyens de consommation, mais à l'acquisition de choses de luxe, et à celle de l'autorité sur les hommes » [Mauss, 19681969 (1914), p. 111]. Les cuivres ne sont pas à proprement parler de la monnaie, mais ils en sont venus avec l'intégration de l'économie indienne dans l'économie globale canadienne «à jouer le même rôle dans leur société que la monnaie dans la nôtre, comme le souligne Godelier à propos des objets précieux en Mélanésie, du moins lorsque celle-ci fonctionne comme capital, c'est-à-dire lorsque, investie dans certaines transactions commerciales, industrielles ou autres, elle produit une valeur en plus, une valeur qui vient s'ajouter à celle investie au départ. Bref, la monnaie fonctionne comme capital lorsqu'elle crée du profit, lorsqu'elle est une richesse qui produit de la richesse » [Godelier, 1984, p. 82, voir aussi 1999, p. 88]. Le cuivre est investi d'une valeur spéculative par la médiation matérielle des couvertures. La véritable monnaie, c'est la couverture. Pour que soient réunies les conditions d'une augmentation de capital, il faut disposer d'un substitut du cuivre qui puisse à la fois circuler à sa place et permettre d'en évaluer la valeur : c'est la couverture qui dans ce contexte est d'emblée un instrument d'usage contradictoire car il est disponible en quantité illimitée, alors que les cuivres existent en quantité finie. La couverture est une monnaie qui n'est pas au fondement d'un système économique autonome. Dans la pratique, les choses se passent ainsi : le cuivre et la couverture sont deux « aspects » distincts d'une même réalité, mutuellement convertibles et relevant de la même sphère de pouvoir.

Dans certains cas, les couvertures pouvaient être prêtées à intérêt ${ }^{32}$ en vue de faire fructifier un capital. Au sens propre du terme, un lot de couvertures est un stock que l'on peut faire « travailler », ce n'est pas seulement une accumulation neutre. On retrouve là une caractéristique qui est à certaines différences près commune au cuivre et à la couverture. La couverture n'est pas un objet inerte, son activité n'est pas proprement liée à celle d'un marché, mais à un jeu d'interactions qui a pour cadre les pratiques cérémonielles et la quête de prestige des chefs. C'est ici qu'il faut introduire la notion de «business potlatch », qui est directement associée au système de capitalisation. Les enrichissements rapides permettant l'entrée

32. Il semblerait que le système de prêt ait été introduit chez les Kwakiutl par un employé du poste de traite de Fort Rupert travaillant pour le compte de la Compagnie de la baie d'Hudson, qui avait trouvé là un moyen d'augmenter ses revenus. Le système de prêt fut accepté sans difficulté par les Kwakiutl, car ceux-ci ont affecté au prêt à intérêt une fonction similaire à celle de la dette qu'engendre le don compensé dans le temps par un contre-don, de valeur égale ou sensiblement supérieure [voir Drucker et Heizer, 1967, p. 78]. 
par effraction dans le système hiérarchique et alimentant la compétition pour le prestige et l'honneur procèdent de ce qu'il est donc convenu d'appeler chez les Kwakiutl le «business potlatch ${ }^{33} »$, mode d'investissement et de financement fondé sur une utilisation habile du crédit et de l'endettement en vue de procéder à des distributions somptuaires. Ce sont précisément ces nouvelles modalités d'accumulation de biens, introduites à la fin du XIX ${ }^{\mathrm{e}}$ siècle, qu'a décrites Boas, mais en omettant de souligner que don et contre-don, d'une part, prêt à intérêt et remboursement de la dette, d'autre part, étaient deux phénomènes distincts ${ }^{34}$. Le principe fondamental du potlatch, écrit-il, est « d'investir des richesses rapportant des intérêts. Les couvertures de la Compagnie de la baie d'Hudson sont une monnaie d'échange - un étalon de mesure évaluée à 50 cents. Tout est payé en couvertures ou en biens pouvant être convertis en couvertures » [Boas, 1897, p. 341]. L'irruption massive de couvertures contribue ainsi à développer une forme standardisée d'évaluation de la richesse ; les couvertures ont valeur de numéraire et prennent statut d'équivalent général se substituant aux objets précieux. C'est ainsi que les objets de valeur en viennent à constituer un capital, se délestant ainsi de leur symbolique magico-religieuse, comme si la couverture en tant que bien standardisé avait contribué à « séculariser » le potlatch [Wolf, 1999, p. 122]. Mais les conséquences de cette situation ne sont pas circonscrites à ce seul domaine de la sécularisation ; l'intrusion de la monnaie sous la forme particulière des couvertures dérègle le système en favorisant la spéculation dans le double contexte de la vacance de positions au sein du système hiérarchique et de la mise en place du crédit. Il vient un temps où le crédit n'est plus contrôlable : les prêts sont de plus en plus importants et les partenaires de plus en plus nombreux, les créances ne sont plus remboursées et les objets - cuivres ou couvertures - circulent sur simple engagement de crédit ${ }^{35}$. Les spéculateurs prêtent à perte, les mauvais payeurs se dérobent à leurs obligations [Drucker et Heizer, 1967, p. 77 ; Mauzé, 1989, p. 1246]. Les conditions sont réunies pour que cette fuite en avant provoque l'effondrement du système. Ce qui ne manque pas d'arriver.

S'opère ici une disjonction entre deux notions fondamentales : d'une part, une rareté ou plus précisément une existence des cuivres en nombre limité au point qu'à tout moment donné de la vie d'un groupe on peut en faire un inventaire [voir par exemple Hunt 1923] et donc d'une manière ou d'une autre en apprécier la valeur et, d'autre part, la nature de l'unité de compte qui permet d'apprécier

33. J'insiste sur le fait que ce système de financement a été pratiqué à cette échelle exclusivement chez les Kwakiutl. Le modèle kwakiutl est remarquable mais non représentatif des sociétés de la côte Nord-Ouest.

34. On peut se reporter notamment à [Curtis, 1914 ; Drucker et Heizer, 1967 ; Oberg, 1973 ; voir aussi Mauzé, 1986].

35. Chez les Kwakiutl on pouvait acquérir un cuivre en payant un acompte, opération dénommée « faire un oreiller pour le cuivre » [Boas, 1897, p. 345].

36. J'ai décrit ce système à propos des modalités de circulation de canoës fictifs dans les potlatch des Lekwiltoq (une des tribus kwakiutl) au tournant du XXe siècle [Mauzé, 1989]. 
quantitativement cette valeur. En effet, nous sommes dans une situation hautement paradoxale que l'on pourrait présenter ainsi : des objets dont la valeur est raisonnablement spéculative se trouvent en situation d'être confrontés à une unité de mesure infiniment spéculative. La couverture cesse d'être l'étalon qui permet d'apprécier l'importance du cuivre pour acquérir une autonomie d'existence indépendante de l'objet qu'elle est censée apprécier. Il y a un déséquilibre comparable à celui que créerait la rareté d'une marchandise dans un contexte de surabondance de numéraire. À ce moment-là, le devenir du numéraire acquiert un statut autonome de plus en plus indépendant de ce dont il est comptable. Il y a crise parce que le risque est soit que l'objet évaluable perde toute valeur, soit que la notion de valeur de l'objet perde tout sens. La situation est « financièrement » sans issue. Pour reprendre le langage d'Aristote, nous ne sommes plus dans l'économique, fondé sur la recherche d'une adéquation entre une chose et l'étalon qui permet d'en apprécier la valeur, mais dans la chrématistique « du fait de laquelle il semble n'y avoir nulle borne à la richesse et à la propriété » [1993, p. 115 sq.], c'est ce qui se produit avec la surabondance sans justification comptable des couvertures. En quelque sorte, pour reprendre aussi bien le langage d'Aristote que celui de Jacques Derrida [1991, p. 200] qui s'en inspire, le potlatch sous sa forme habituellement convenue est adéquat à ses modalités traditionnelles et au cadre dans lequel ces modalités sont mises en œuvre. Tandis qu'il y a impossibilité de pérenniser l'institution quand l'un des deux éléments, à savoir le mode d'appréciation quantitatif, devient incontrôlable, c'est-à-dire ne relève plus d'une logique inhérente aux enjeux économiques et politiques du groupe. Il n'y a plus «monétarisation » du potlatch, mais « potlatchisation ${ }^{37} »$ - déjà annoncée par les premiers excès du «business potlatch »-du numéraire. Dans cet emballement de la logique économique du potlatch, on comprend que puisse intervenir une spéculation sur des objets virtuels, par exemple des canoës fictifs, dans la simple mesure où la circulation des couvertures n'est plus fondée sur une logique économique rationnelle. Dans ce système où le potlatch était un instrument de pouvoir, la crise économique que l'on vient d'évoquer a pour conséquence un effondrement du système d'autorité.

37. C'est en fait ce qu'explique Boas et que Mauss reprend en soulignant que c'est précisément dans ce texte de 1899 que Boas donne la meilleure explication du fonctionnement du potlatch : "Contracter des dettes d'un côté, payer des dettes de l'autre côté, c'est le potlatch. Ce système économique s'est développé à un tel point que le capital possédé par tous les individus associés de la tribu excède de beaucoup la quantité des valeurs disponibles qui existe ; autrement dit, les conditions sont tout à fait analogues à celles qui prévalent dans notre société à nous : si nous désirons nous faire payer de toutes nos créances, nous trouverions qu'il n'y a à aucun degré assez d'argent, en fait, pour les payer. Le résultat d'une tentative de tous les créanciers de se faire rembourser leurs prêts, c'est une panique désastreuse dont la communauté met longtemps à se guérir » [Boas, 1899 cité par Mauss [1950 (1925), p. 198]. 
AppaduraI, Arjun [éd.] (1986), The Social Life of Things. Commodities in a Cultural Perspective, Cambridge University Press, Cambridge.

ArIStote (1993), Les Politiques, traduction inédite, introduction, bibliographie, notes et index par Raymond Pellegrin, deuxième édition, Flammarion, GF, Paris.

BarnetT, Homer G. (1938), « The Nature and the Function of the Potlatch », American Anthropologist, 40, p. 349-358.

BoAs, Franz (1897), « The Social Organization and the Secret Societies of the Kwakiutl Indians », Report of the U.S. National Museum for 1895, Washington (Johnson Reprint, New York, 1970).

BoAs, Franz (1899), « Twelfth And Final Report on the North-Western Tribes of Canada », Report of the British Association for the Advancement of Science for 1898, Londres.

BoAs, Franz (1921), «Ethnology of the Kwakiutl Indians, Bureau of the American Ethnology », 35th Annual Report, parts 1 and 2. Washington, D. C., Government Printing Office.

BoAs, Franz (1966), Kwakiutl Ethnography, édité par Helen Codere, Chicago University Press, Chicago.

BoAs, Franz et George Hunt (1905), Kwakiutl Texts, Publications of the North Jesup Expedition, vol. 3, Leiden.

Boyd, Robert (1999), The Coming of the Spirit of Pestilence : Introduced Infectious Diseases and Population Decline among Northwest Coast Indians, 1774-1874, UCB Press Seattle, University of Washington Press, Vancouver.

Codere, Helen (1950), Fighting with Property : A Study of Potlatching and Warfare, 1792-1930, University of Washington Press, Seattle.

- (1961), « Kwakiutl », in Edward H. SPICER (éd.), Perspectives in American Indian Cultural Change, Chicago University Press, Chicago, p. 431-516.

CuRTIS, Edward (1915), The North American Indian, vol. 10. The Kwakiutl, Plimpton Press, Nordwood, Mass..

De Laguna, Frederica (1972), Under Mount Saint Elias : The History and Culture of the Yakutat Tlingit, 3 vol., Smithsonian Contributions to Anthropology, Washington.

DERrIDA, Jacques (1991), Donner le temps. 1. La fausse monnaie, Galilée, Paris.

Donald, Leland (1997), Aboriginal Slavery on the Northwest Coast of North America, University of California Press, Berkeley.

Duff, Wilson (1959), ms. Notes on « Potlatch and Feast », Royal British Columbia Museum, Victoria.

- (1981), « The Killer Whale Copper », in Donald Аввотт (éd.), The World is as Sharp as a Knife. An Anthology in Memory of Wilson Duff, B. C. British Columbia Provincial Museum, Victoria, p. 153-156.

DrUCKER, Philip et HeIZER Robert F. (1967), To Make my Name Good. A Reexamination of the Southern Kwakiutl Potlatch, University of California Press, Berkeley.

Emmons, George T. (1991), The Tlingit Indians, édition revue et augmentée par Frederica de Laguna, University of Washington Press, Seattle, American Museum of Natural History, New York.

FISHER, Robin (1977), Contact and Conflict : Indian-European Relations in British Columbia, 1774-1890, University of British Columbia Press, Vancouver.

Garfield, Viola E. (1939), Tsimshian Clan and Society, The University of Washington (University of Washington Publications in Anthropology, 7.3), Seattle. 
GoDELIER, Maurice (1994), « Monnaies et richesses dans divers types de société et leur rencontre à la périphérie du capitalisme », Actuel Marx, $15, \mathrm{n}^{\circ}$ spécial «L'inconscient du social », p. 77-97.

- (1996), L'Énigme du don, Fayard, Paris.

Goldman, Irvin (1975), The Mouth of Heaven : An Introduction to Kwakiutl Religious Thought, John Wiley and Sons, New York.

Gregory, C. A. (1982), Gifts and Commodities, Academic Press, Londres.

HARKIN, Michael (1997), The Heiltsuks. Dialogues of Culture and History on the Northwest Coast, University of Nebraska, Lincoln.

HaYman, John [éd.] (1989), Robert Brown and the Vancouver Island Exploring Expedition, University of British Columbia Press, Vancouver.

HunT, George (1923), History of 20 Coppers from Alert Bay, B. C., Archives of British Columbia, Victoria.

KAN, Sergei (1989), Symbolic Immortality. The Tlingit Potlatch of the Nineteenth Century, Smithsonian Institution Press, Washington et Londres.

KNIGHT, Rolf (1978), Indians at Work : An Informal History of Native Indian Labour in British Columbia, 1858-1930, New Star Books, Vancouver.

KeIthan, E. L. (1964), « Origin of the "Chiefs'Coppers" or “Tinneh" », Anthropological Papers of the University of Alaska, 12 (2), p. 59-78.

Kobrinsky, Vernon (1975), « Dynamics of the Fort Rupert Class Struggle : Fighting with Property Vertically Revisited », in Vernon SERL et Herbert TAYLOR (éd.), Papers in Honour of Harry Hawthorn, Western Washington State College, Bellingham, p. 3259.

Krause, Aurel (1956), The Tlingit Indians, traduction Erna Gunther, University of Washington Press, Seattle.

LÉVI-STRAUSS, Claude (1979), La Voie des masques, édition revue et augmentée de trois excursions, Plon, Paris.

Lisiansky, capitaine Urey (1814), A Voyage Around the World, 1803, 1804, 1805, 1806, imprimé pour J. Booth, Edinburgh.

MaCDonald, George (1981), « Cosmic Equations in Northwest Coast Indian Art », in Donald Аввотт (éd.), The World is as Sharp as a Knife. An Anthology in Memory of Wilson Duff, British Columbia Provincial Museum, Victoria, B. C., p. 225-238.

- (1996), Haida Art, University of Washington Press, Seattle.

MASCO, Joseph (1995), " "It is a Strict Law that Bids us Dance" : Cosmologies, Colonialism, Death, and Ritual Authority in the Kawkwaka'waks Potlatch, 1849 to 1922 », Comparative Studies in Society and History, 37, p. 41-75.

Mauss, Marcel (1914), «Les origines de la notion de monnaie », Anthropologie, 25, réédité in Euvres, 1958-1969, vol. 2, Minuit, Paris, p. 106-112.

- (1925), «Essai sur le don. Forme et raison de l'échange dans les sociétés archaïques », Année sociologique, ns 1923-1924, réédité en 1950 in Sociologie et anthropologie avec une introduction de Claude Lévi-Strauss, Presses universitaires de France, Paris.

MAUZÉ, Marie (1986), «Boas, les Kwagul et le potlatch. Éléments pour une réévaluation », L'Homme, $\mathrm{n}^{\circ} 100$, p. 21-63.

- (1987), «Georges Bataille et le potlatch : à propos de La Part maudite », in Écrits d'ailleurs. Georges Bataille et les ethnologues, textes réunis par Dominique Lecocq et Jean-Luc Lory, Éditions de la Maison des Sciences de l'Homme, Paris, p. 31-38.

- (1989), «Le canoë dans le potlatch Lekwiltoq », L’Homme, n 109, p. 117-128.

- (1995), «Potlatching as Ever », European Review of Native American Studies, 9 (2), p. 25-31. 
- (1996), «Commentaire : "Le potlatch ou l'invention d'un concept anthropologique" ", Serge GruzinsKi et Nathan WaChTEL, (éd.), Le Nouveau Monde-Mondes nouveaux. L'expérience américaine, Éditions Recherche sur les civilisations/Éditions de l'EHESS, Paris, p. 699-702.

McIlwaith T.F. (1992), The Bella Coola Indians, avec une introduction de John Barker, University of Toronto Press, Toronto.

NASH, Manning (1966), Primitive and Peasant Economic Systems, Chandler Press, San Francisco.

NiBlaCK, Albert (1890), « The Coast Indians of Southern Alaska and Northern British Columbia », Annual Report of the U.S. National Museum for 1888, Washington.

OBERg, Kalervo (1973), The Social Economy of the Tlingit Indians, University of Washington Press, Seattle.

Olson, Ronald (1933), «Clan and Moiety in Native America », University of California Publications in Archeology and Ethnology 33 (4), p. 351-421.

QuigGin, A.H. (1970) [1949], A Survey of Primitive Money, avec une introduction de A. C. Haddon, Methuen Library Reprints, Londres.

REID, Martine (1986), « The Significance of Colour among the Kwagiulth », in Doreen Jensen et Polly Sargent, Robes of Power. Totem Poles on Cloth, The University of British Columbia Press, Vancouver, p. 76-77.

SAHLINS, Marshall (1988), «Cosmologies of Capitalism : the Trans-Pacific Sector of the "World System" », Proceeding of the British Academy LXXIV, p. 1-51.

- (1999), Les Lumières en anthropologie ?, Société d'ethnologie, Nanterre.

SCHULte-Tenckhoff, Isabelle (1986,) Potlatch : conquête et invention. Réflexion sur un concept anthropologique, Éditions d'en bas, Lausanne.

SEwID-SMith, Daisy (1986), « Kwagiulth Ceremonial Blankets », in Doreen Jensen et Polly Sargent, Robes of Power. Totem Poles on Cloth, The University of British Columbia Press, Vancouver, p. 63-64.

WaLens, Stanley (1981), Feasting with Cannibals : An Essay on Kwakiutl Cosmology, Princeton University Press, Princeton, N. J.

Waterman, T. T. (1932), « Some Conundrums in Northwest Coast Art », American Anthropologist 25 (4), p. 435-451.

WeINER, Annette B. (1985), «Inalienable Wealth», American Ethnologist 12 (2), p. 210227.

- (1992), Inalienable Possessions : The Paradox of Keeping-while-Giving, University of California Press, Berkeley, 1992.

Widerspach-Thor Martine, de (1981), « The Equation of the Copper », in Donald N. Аввотт (éd.), The World is as Sharp as a Knife : An Anthology in Honour of Wilson Duff, British Columbia Provincial Museum, Victoria, p. 157-174.

Wingert, P. S. (1949), American Indian Sculpture. A Study of the Northwest Coast Art, J. J. Augustin, New York.

Wolf, Eric (1999), Envisioning Power. Ideologies of Dominance and Crisis, University of California Press, Berkeley. 